ARTICLE

Received 6 Sep 2012 | Accepted 3 Jan 2013 | Published 12 Feb $2013 \quad$ DOl: 10.1038/ncomms2442

\title{
Universal current-velocity relation of skyrmion motion in chiral magnets
}

Junichi Iwasaki ${ }^{1}$, Masahito Mochizuki ${ }^{1} \&$ Naoto Nagaosa ${ }^{1,2}$

Current-driven motion of the magnetic domain wall in ferromagnets is attracting intense attention because of potential applications such as racetrack memory. There, the critical current density to drive the motion is $\sim 10^{9}-10^{12} \mathrm{~A} \mathrm{~m}^{-2}$. The skyrmions recently discovered in chiral magnets have much smaller critical current density of $\sim 10^{5}-10^{6} \mathrm{Am}^{-2}$, but the microscopic mechanism is not yet explored. Here we present a numerical simulation of Landau-Lifshitz-Gilbert equation, which reveals a remarkably robust and universal currentvelocity relation of the skyrmion motion driven by the spin-transfer-torque unaffected by either impurities or nonadiabatic effect in sharp contrast to the case of domain wall or spin helix. Simulation results are analysed using a theory based on Thiele's equation, and it is concluded that this behaviour is due to the Magnus force and flexible shape-deformation of individual skyrmions and skyrmion crystal, which enable them to avoid pinning centres.

\footnotetext{
${ }^{1}$ Department of Applied Physics, The University of Tokyo, 7-3-1, Hongo, Bunkyo-ku, Tokyo 113 8656, Japan. ${ }^{2}$ Cross-Correlated Materials Research Group (CMRG), and Correlated Electron Research Group (CERG), RIKEN-ASI, Wako, Saitama 351 0198, Japan. Correspondence and requests for materials should be addressed to N.N. (email: nagaosa@ap.t.u-tokyo.ac.jp).
} 
$t$ has long been recognized that the spin-polarized electric current can drive the motion of spin textures via the spintransfer-torque since the original theoretical proposals ${ }^{1,2}$. Subsequent experimental demonstrations of this effect using the spin-valve systems and the ferromagnetic domain walls have stimulated recent active researches ${ }^{3,4}$. However, the Joule heating has been a serious issue because a large current density $j$ is necessary to overcome the pinning, and, therefore, the experiments usually have been done using a short pulse of electric current. The current-velocity relation of the domain-wall motion has been well studied ${ }^{3-9}$, which is sensitive to the impurity pinning, the Gilbert damping, and nonadiabatic effects.

Skyrmion $^{10}$, a vortex-like topological spin texture where the spins point in all directions wrapping a sphere, is recently discovered in chiral-lattice magnets with the DzyaloshinskiiMoriya (DM) interaction such as $\mathrm{MnSi}, \mathrm{Fe}_{1-\mathrm{x}} \mathrm{Co}_{\mathrm{x}} \mathrm{Si}$ and FeGe. The DM interaction naturally leads to a helical spin order (HL), which turns into a triangular skyrmion crystal (SkX) under an external magnetic field $\boldsymbol{B}$ (refs 11, 12) as observed in a narrow region of the $B-T$ phase diagram for bulk samples by neutronscattering experiments ${ }^{13}$. Enhanced stability of the SkX in twodimensional (2D) systems or in thin-plate samples was predicted theoretically ${ }^{14,15}$, and was indeed confirmed by Lorentz microscope experiments ${ }^{16,17}$. Figure 1 shows a phase diagram at $T=0$ as well as schematic pictures of spin configurations in the $\mathrm{HL}, \mathrm{SkX}$ and ferromagnetic phases in the $2 \mathrm{D}$ system. It was experimentally demonstrated that ultralow current density of $\sim 10^{5}-10^{6} \mathrm{~A} \mathrm{~m}^{-2}$, orders of magnitude smaller than the current density typically used for the domain-wall manipulation, can drive translational and rotational motions of SkX domains in MnSi (ref. 18) and FeGe (ref. 19). This finding has opened a new route to low-power manipulation of magnetic structures. However, experimental knowledge about the microscopic dynamics of skyrmions is very limited, and the origin of the very small critical current has not been explored.

Under this circumstance, theoretical clarification of the current-driven skyrmion dynamics becomes an issue of vital importance, which we address in this paper. We report the universal relation between the current density and the velocity, which does not hold in the case of HL or the ferromagnetic domain wall. We have also discovered the peculiar motion of the skyrmions characterized by flexible shape-deformation of individual skyrmions and SkX so as to avoid pinning centres.

\section{Results}

Model and its simulation. The spin system in thin-plate samples of chiral-lattice magnets is described by a classical Heisenberg model on the $2 \mathrm{D}$ square lattice, where the local magnetic moments $\boldsymbol{M}_{\boldsymbol{r}}$ defined as $\boldsymbol{M}_{\boldsymbol{r}} \equiv-\boldsymbol{S}_{\boldsymbol{r}} / \hbar\left(\boldsymbol{S}_{\boldsymbol{r}}\right.$ represents the local spin at $\boldsymbol{r}$ ) are treated as classical vectors whose length is fixed to be

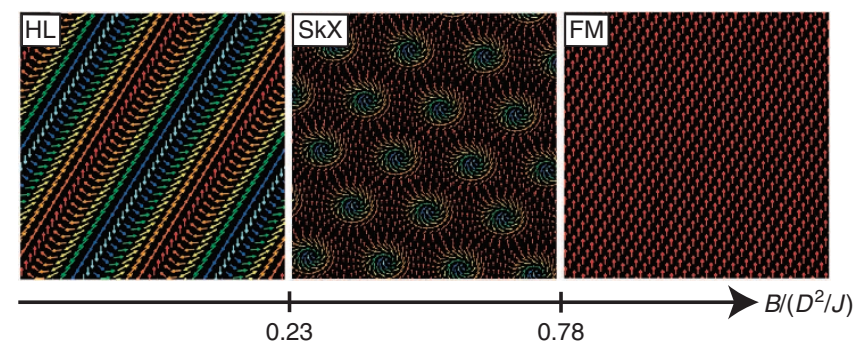

Figure 1 | Spin textures in chiral-lattice magnets. Phase diagram of the Hamiltonian (1) with $D / J=0.18$ at $T=0$ as a function of magnetic field $B$ in the absence of impurities where schematic spin configurations in the helical $(\mathrm{HL})$, skyrmion crystal $(\mathrm{SkX})$ and ferromagnetic $(F M)$ phases are displayed.
$\left|\boldsymbol{M}_{\mathbf{r}}\right|=M$. The model includes the ferromagnetic-exchange interaction, the DM interaction, and the Zeeman coupling. We also consider the effect of impurities by introducing the magnetic anisotropy with easy magnetization axis perpendicular to the $2 \mathrm{D}$ plane on the randomly distributed impurity sites. The Hamiltonian is given by

$$
\begin{aligned}
\mathscr{H}= & -J \sum_{\boldsymbol{r}} \boldsymbol{M}_{\boldsymbol{r}} \cdot\left(\boldsymbol{M}_{\boldsymbol{r}_{+} e_{x}}+\boldsymbol{M}_{\boldsymbol{r}_{+} e_{y}}\right) \\
& -D \sum_{\boldsymbol{r}}\left(\boldsymbol{M}_{\boldsymbol{r}} \times \boldsymbol{M}_{\boldsymbol{r}+e_{x}} \cdot \boldsymbol{e}_{x}+\boldsymbol{M}_{\boldsymbol{r}} \times \boldsymbol{M}_{\boldsymbol{r}+e_{y}} \cdot \boldsymbol{e}_{y}\right) \\
& -\boldsymbol{B} \cdot \sum_{\boldsymbol{r}} \boldsymbol{M}_{\boldsymbol{r}}-A \sum_{\boldsymbol{r} \in I} \boldsymbol{M}_{z \boldsymbol{r}}^{2} .
\end{aligned}
$$

Here $I$ denotes positions of the impurities. For the parameters, we adopt realistic values as $J=1 \mathrm{meV}, D / J=0.18$ and $A / J=0.2$. The exchange interaction of $J=1 \mathrm{meV}$ gives the critical temperature $T_{\mathrm{c}} \sim 60 \mathrm{~K}$ for the paramagnetic-helimagnetic transition comparable to the experimental $T_{\mathrm{c}}$ for $\mathrm{MnSi}(\sim 30 \mathrm{~K})$ and $\mathrm{Fe}_{1-\mathrm{x}} \mathrm{Co}_{\mathrm{x}} \mathrm{Si}(\sim 300 \mathrm{~K})$. The ratio $\mathrm{D} / \mathrm{J}$ determines the size of skyrmion $\xi$, and $D / J=0.18$ reproduces the diameter of 50 sites, which corresponds to $\xi=25 \mathrm{~nm}$ when the typical lattice parameter $a=5 \AA$ is assumed. This value is comparable to the experimentally observed sizes, that is, $\xi=18 \mathrm{~nm}$ for $\mathrm{MnSi}$. The strength of magnetic anisotropy at impurity sites, $A / J=0.2$, turns out to give a critical current of $j_{\mathrm{c}} \sim 10^{10}-10^{11} \mathrm{~A} \mathrm{~m}^{-2}$ for the current-driven motion of the HL texture, which reproduces the experimental value observed for the ferromagnetic domain-wall motion $^{3-5}$. The magnetic field $\boldsymbol{B}=(0,0, B)$ is applied normal to the plane where $B / J=-1.5 \times 10^{-2}$ for the SkX phase, and $B=0$ for the HL phase. The magnetic field $B$ is measured in energy unit here, and this value of $1.5 \times 10^{-2} \mathrm{meV}$ corresponds to $\sim 0.25$ Tesla. This value is comparable to the strength of the magnetic field at which the SkX is observed in MnSi (ref. 13).

It should be mentioned that the demagnetizing field or the magnetic dipole interaction is neglected in our model, which can be justified for the SkX with lattice spacing $d$ of $10-50 \mathrm{~nm}$ discussed in the present work. In thin-plate magnets, a lattice of circular-shaped magnetic bubbles is often realized by the competition among the magnetostatic energy because of the dipole interaction, the magnetic exchange interaction, and the uniaxial magnetic anisotropy. We can derive the relationship between the bubble lattice spacing $d$ and the sample thickness $l$ by minimizing the total energy of these three contributions ${ }^{20}$, and find that to realize a bubble lattice with $d \sim 10 \mathrm{~nm}$, the sample thickness should be much smaller than $\sim 1 \mathrm{~nm}$ (see Methods). This means that even the single atomic layer is not thin enough. Therefore, the effect of demagnetizing field is negligible compared with the DM interaction in the present chiral-magnet systems.

We study the current-driven spin dynamics at $T=0$ by numerically solving the Landau-Lifshitz-Gilbert (LLG) equation (see Methods):

$$
\begin{aligned}
\frac{\mathrm{d} \boldsymbol{M}_{\boldsymbol{r}}}{\mathrm{d} t}= & -\gamma \boldsymbol{M}_{\boldsymbol{r}} \times \boldsymbol{B}_{\boldsymbol{r}}^{\mathrm{eff}}+\frac{\alpha}{M} \boldsymbol{M}_{\boldsymbol{r}} \times \frac{\mathrm{d} \boldsymbol{M}_{\boldsymbol{r}}}{\mathrm{d} t}+\frac{p a^{3}}{2 e M}(\boldsymbol{j} \cdot \nabla) \boldsymbol{M}_{\boldsymbol{r}} \\
& -\frac{p a^{3} \beta}{2 e M^{2}}\left[\boldsymbol{M}_{\boldsymbol{r}} \times(\boldsymbol{j} \cdot \nabla) \boldsymbol{M}_{\boldsymbol{r}}\right],
\end{aligned}
$$

with $\boldsymbol{B}_{r}^{\text {eff }}=-\frac{1}{\hbar \gamma} \frac{\partial \mathscr{H}}{\partial \boldsymbol{M}_{r}}$. Here $\gamma=g \mu_{\mathrm{B}} / \hbar(>0)$ is the gyromagnetic ratio, $p$ is the spin polarization of the electric current, $e(>0)$ is the elementary charge, and $a$ is the lattice constant. The second term, so-called $\alpha$ term, denotes the Gilbert damping where $\alpha$ is fixed at $\alpha=0.04$. This value is also a typical value for the ferromagnetic metal $^{21-24}$ and the dilute magnetic semiconductors ${ }^{25}$. The third and fourth terms describe the coupling between spins and 
spin-polarized electric current $\boldsymbol{j}$. Microscopically the conductionelectron spins interact with local magnetic moments via the Hund's-rule coupling $J_{\mathrm{H}}$ or the local exchange interaction $J_{\text {sd. }}$. As the spin-polarized electric current has a flux of angular momentum, it works as a torque acting on the magnetic moments in noncollinear spin structures. The third term, socalled spin-transfer-torque term, is derived under the assumption that the conduction-electron spins are always parallel to the local magnetic moments $\boldsymbol{M}_{\boldsymbol{r}}$ owing to $J_{\mathrm{H}}$ and $J_{\mathrm{sd}}($ refs 26,27$)$. Note here that the current-driven spin motion due to the Berry phase discussed in refs 26,27 is equivalent to the spin-transfer-torque in equation (2). On the other hand, the fourth term, so-called $\beta$ term, describes the coupling between spin-polarized current and local magnetizations owing to nonadiabatic effects. Four different values of $\beta$ are examined, that is, $\beta=0,0.5 \alpha, \alpha$ and $2 \alpha$.

In this work, the current-driven motions of both SkX and HL textures are studied because characteristics and advantages of the skyrmion against the ferromagnetic domain wall can be highlighted by comparing these two cases. We find a much smaller critical current and less influence of impurities for the $\mathrm{SkX}$ case. Here, the HL texture can be regarded as successively arranged ferromagnetic Bloch walls where the simulated currentdriven motion turns out to be well described by analytically derived equations for a single domain-wall motion. This means that the reason for low critical current of SkX relative to that of the ferromagnetic domain wall is not due to the fact that the spintransfer-torque acts all over the sample due to the periodic structures. It should also be mentioned that the present system is appropriate for such comparison because we can produce both SkX and HL states only by varying the external magnetic field $B$ without any changes in other parameters.

Relation between the current density and the velocity. Figure $2 \mathrm{a}$ shows calculated velocities $v_{\|}$(parallel to $\boldsymbol{j}$ ) as functions of the current density $j$ for the $\mathrm{HL}$ and $\mathrm{SkX}$ phases with different values of $\beta$. We examine the clean case without impurity $(x=0)$ and the dirty case with impurities $(x=0.1 \%)$ where $x$ is the impurity concentration. The most remarkable fact here is that the $j-v_{\|}$ characteristics in the SkX phase (blue data points) remains universal, independent of the Gilbert damping $\alpha$, nonadiabatic effect $\beta$ and impurities. This property is in accord with the expression $v_{\|}=\frac{p a^{3}}{2 e M} j$ expected in the spin-transfer-torque mechanism. Figure $2 \mathrm{~b}$ magnifies Fig. $2 \mathrm{a}$ in the low $j$ region, where the advantage of the skyrmion relative to the domain wall or the $\mathrm{HL}$ is stressed. The suppression of the velocity $v_{\|}$by impurities cannot be identified within the accuracy of this figure, manifesting the robustness of the universal relation. This indicates that the SkX is an ideal system for manipulation via the spin-transfer-torque mechanism down to a very low current density.

In contrast, the $j-v_{\|}$characteristics in the HL phase is quite sensitive to all these three factors as seen in Fig. 2a, which are similar to those of the single ferromagnetic domain wall ${ }^{7}$. In the HL phase, the intrinsic pinning prevents the motion at $\beta=0$, while the relation $v_{\|} \propto(\beta / \alpha) j$ holds without impurities. The impurity pinning suppresses the velocity $v_{\|}$, and gives rise to a finite critical current density $j_{\mathcal{C}}$, which is of the order of $10^{10}-10^{11} \mathrm{~A} \mathrm{~m}^{-2}$ when $x=0.1 \%$ in the present model. This value of $x$ corresponds to a rather high density of impurities if not intentionally doped. Note that the current density that we focus on here is much smaller than that typically used for the racetrack memory in $\mathrm{Py}^{4}$. There, the velocity of $v_{\|} \sim 110 \mathrm{~m} \mathrm{~s}^{-1}$ at a current density of $j \sim 1.5 \times 10^{12} \mathrm{~A} \mathrm{~m}^{-2}$ is discussed and the domain wall has an advantage over skyrmions in that region especially with $\beta=2 \alpha$ for Py. Here, these values of $v_{\|}$and $j$ are semiquantitatively consistent with the line for HL with $\beta=2 \alpha$ in
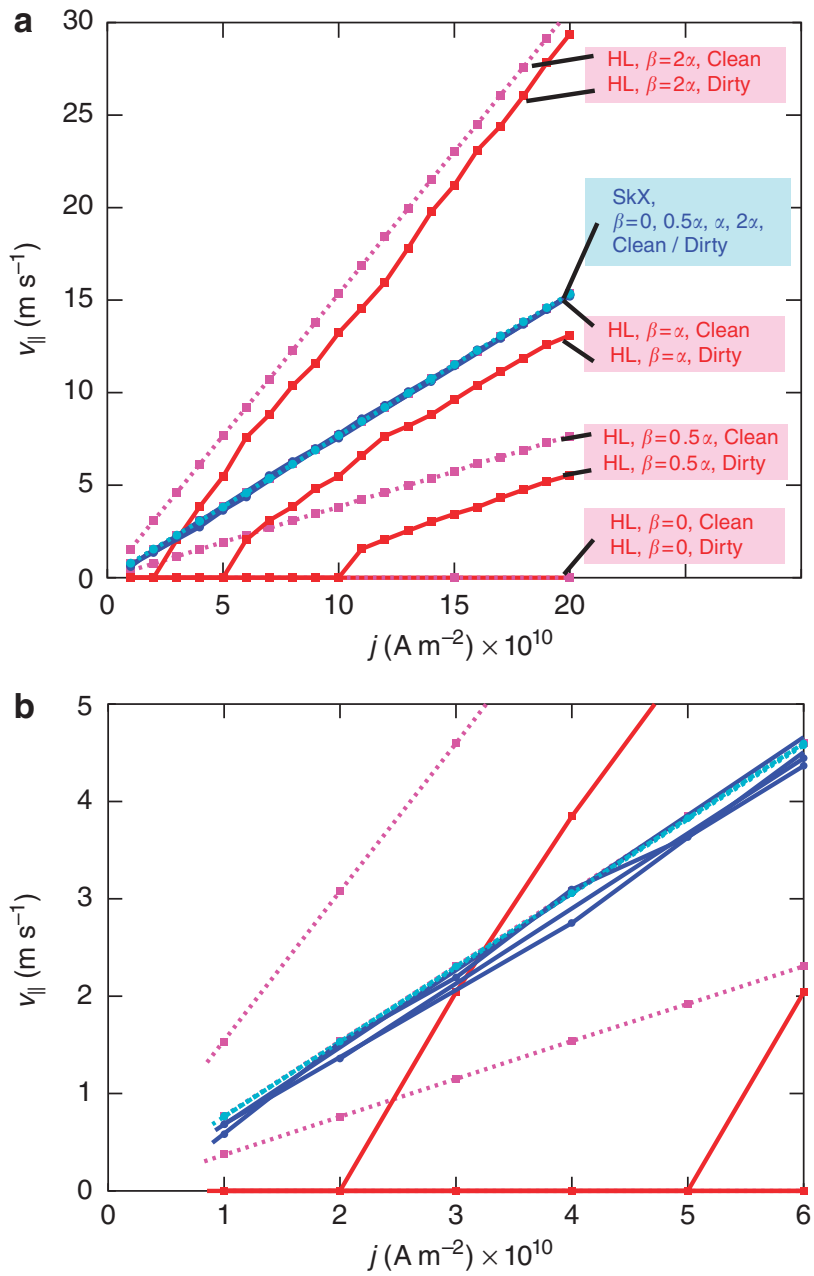

Figure 2 | Velocities of the current-driven motions. (a) Longitudinal velocities $v_{\|}$of the current-induced motions of the helical $(\mathrm{HL})$ and skyrmion crystal (SkX) phases as functions of the current density $j$ for several values of $\beta$. Concerning the impurity effects, the clean case without impurity $(x=0)$ and the dirty case with impurities $(x=0.1 \%)$ are examined where $x$ is the impurity concentration. Lines for the SkX are all identical and overlapped within the accuracy of the numerical simulation, irrespective of the presence or absence of nonadiabatic effects (the $\beta$ term) and impurities. (b) Magnification of Fig. 2 (a) in the region of low current density.

Fig. 2a. Skyrmions will be useful for ultralow-energy cost operation with a moderate speed $\left(\sim 1-10 \mathrm{~m} \mathrm{~s}^{-1}\right)$, which is complementary to the domain-wall motion. The energy dissipation per time is proportional to $j^{2}$, while the time to manipulate the fixed amount of information is inversely proportional to $j$. Therefore, the energy cost to manipulate the same amount of information is proportional to the current density $j$.

The contrasting $\beta$-dependence between the SkX and HL cases comes from difference of the relevant variables for their currentdriven motions. Namely, in the case of HL or ferromagnetic Bloch walls, the spin rotation angle within the plane parallel to the domain wall and the spin component perpendicular to it are canonically conjugate. Therefore, the motion associated with spin rotation is accompanied by the spin tilting out of the plane, and hence costs magnetic anisotropy energy. This results in the finite threshold current. Such an intrinsic pinning effect is most remarkable when $\beta=0$, but becomes loosen for the finite 
$\beta$, which gives the strong sensitivity to the value of $\beta$ for the current-driven motion of HL and domain walls. In the case of skyrmions, the relevant variables are the coordinates of the centre of skyrmion, and hence the intrinsic pinning does not work. This leads to the $\beta$-insensitive behaviour of the current-driven motion of SkX.

Spin configurations during the motion. The effects of impurities are directly seen in the dynamical spin configurations during the motion. In Fig. 3, we display snapshots of magnetizations in the moving HL $(\mathrm{a}, \mathrm{b})$ and $\operatorname{SkX}(\mathrm{c}, \mathrm{d}, \mathrm{e})$. The impurity positions are indicated by green dots (See also Supplementary Movies 1 and 2). Note that even with impurity concentration of $x=0.1 \%$, the characteristic length scale of the distortion is longer than the mean distance between impurities, and hence the collective pinning picture can be applied here ${ }^{28,29}$. It should also be mentioned that because of the line-shaped spin alignments, the moving HL cannot avoid passing over impurities, so that the impurities are
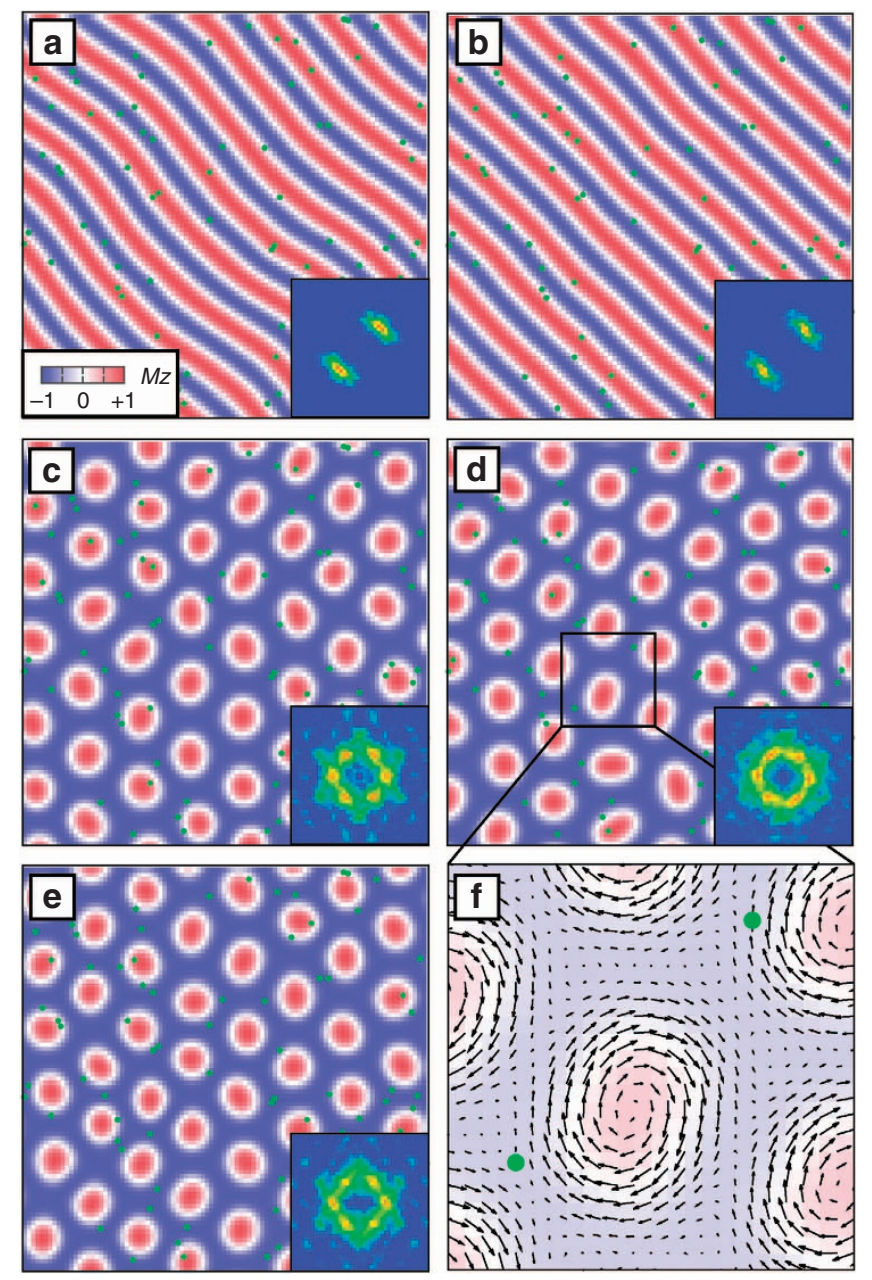

Figure 3 | Simulated current-driven motions of spin textures. Snapshots of the dynamical spin configurations at selected times: (a), Helical phase $(\mathrm{HL})$ at $t=4.55 \times 10^{-8} \mathrm{~s},(\mathbf{b}), \mathrm{HL}$ at $t=4.87 \times 10^{-8} \mathrm{~s},(\mathbf{c})$, Skyrmion crystal $(\mathrm{SkX})$ at $t=1.30 \times 10^{-8} \mathrm{~s},(\mathbf{d}), \mathrm{SkX}$ at $t=2.60 \times 10^{-8} \mathrm{~s}$, and (e), $\mathrm{SkX}$ at $t=4.87 \times 10^{-8} \mathrm{~s}$. (f), Magnified view of (e) in which skyrmions distorted from their original circular shape can be seen. The numerical simulation was performed for $\beta=\alpha$ with the current density $j=6.0 \times 10^{10} \mathrm{~A} \mathrm{~m}^{-2}$ for $\mathrm{HL}$ and $j=4.0 \times 10^{10} \mathrm{~A} \mathrm{~m}^{-2}$ for SkX in the presence of impurities with $x=0.1 \%$. Positions of the impurities are indicated by green dots. always active, and distort the HL spin structure. The motion of the HL is intermittent, that is, sometimes pinned by the impurities and slow down (Fig. 3a), and sometimes depinned and moves quickly (Fig. 3b).

The motion of the SkX is completely different from that of the HL as shown in Fig. 3c-f. Skyrmions in the moving SkX can avoid being trapped by impurities through distorting the triangular lattice and deforming the shape of individual skyrmions. Furthermore, each skyrmion as a particle-like object can wind its trajectory to avoid impurities owing to the fact that the $X$ and $Y$ coordinates of the skyrmion core is canonical conjugate because of the spin Berry phase term, and it rotates around the attractive centre (Supplementary Movie 3). This dynamics is analogous to that of vortex system. However, the ferromagnetic region between skyrmions, which is essential to avoid the impurities, is absent in the case of vortex crystal. The shape of the SkX temporally changes, and it is distorted rather significantly in Fig. 3d, while rather weakly in Fig. 3c,e. However, the velocity of the SkX is rather steady with much smaller fluctuation compared with the HL. Such kinds of motions are key features of the SkX for explaining their ultralow $j_{c}$.

Time-evolution of the Bragg-peak intensities. Above-predicted dynamical deformation of the SkX could be detected in the timeresolved neutron-scattering experiment. Figure 4 shows simulated temporal change of normalized Bragg-peak intensities of the moving SkX for several values of $j$. The dashed line corresponds to the peak height of perfect SkX without distortion. For finite $x$, the peak height temporally fluctuates indicating the timedependent deformation of the SkX. We find that the fluctuation is less pronounced for a larger $j$. This is because the fast moving skyrmions do not feel the impurity potentials so much. Here the time-scale $t_{0}$ is $\sim 10^{-9} \mathrm{~s}$ for $j \sim 10^{10} \mathrm{~A} \mathrm{~m}^{-2}$ and the recent neutron-scattering experiment can trace the time-evolution in this time-scale ${ }^{30}$. Also the quasi-elastic neutron-scattering spectroscopy in frequency domain will be useful to detect the fluctuation.

\section{Discussion}

At this stage, we discuss the simulated results in the light of theory established in ref. 31 and Supplementary of ref. 32. Using an approach proposed by Thiele ${ }^{33}$, the LLG equation can be mapped onto the translational mode in the continuum limit by

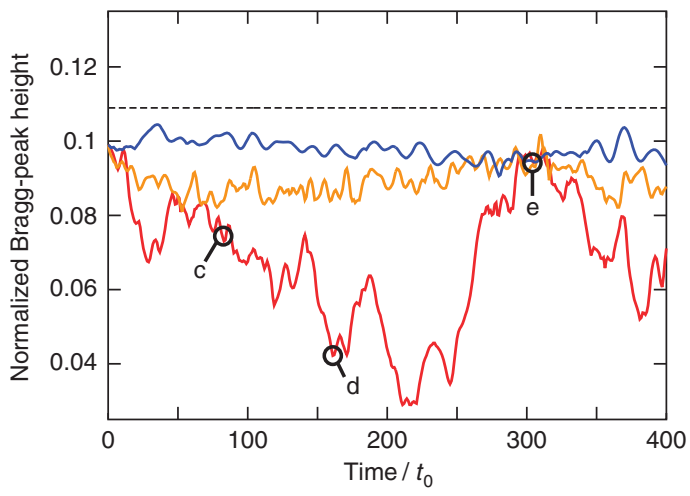

Figure 4 | Time-dependence of the Bragg-peak intensity associated with the motion of skyrmion crystal: The horizontal axis denotes time normalized by $t_{0}=\frac{6.5 \mathrm{Am}^{-2}}{j} \mathrm{~s}$. Dashed line represents the Bragg-peak intensity for the undistorted skyrmion crystal without impurity. The red, orange and blue lines represent the data for $j=4.0 \times 10^{10} \mathrm{~A} \mathrm{~m}^{-2}$, $j=8.0 \times 10^{10} \mathrm{~A} \mathrm{~m}^{-2}$ and $j=2.0 \times 10^{11} \mathrm{~A} \mathrm{~m}^{-2}$, respectively. Open circles with letters indicate corresponding snapshots in Fig. 3. 
assuming the rigidity of spin textures during the drift motion. One obtains 31,32

$$
\boldsymbol{G} \times\left(\boldsymbol{v}_{\mathrm{s}}-\boldsymbol{v}_{\mathrm{d}}\right)+\mathcal{D}\left(\beta \boldsymbol{v}_{\mathrm{s}}-\alpha \boldsymbol{v}_{\mathrm{d}}\right)+\boldsymbol{F}_{\text {pin }}=\mathbf{0},
$$

where $\boldsymbol{v}_{\mathrm{d}}$ is the drift velocity of the spin texture and $\boldsymbol{v}_{\mathrm{s}}=-\frac{p a^{3}}{2 e M} \boldsymbol{j}$ is the velocity of the conduction electrons. The first term in the left hand side of equation (3) is the Magnus force and the second one is the dissipative force. The third one is the phenomenological pinning force due to the impurities ${ }^{31,32}$ :

$$
\boldsymbol{F}_{\text {pin }} \sim-4 \pi v_{\text {pin }} f\left(v_{\mathrm{d}} / v_{\text {pin }}\right) \boldsymbol{v}_{\mathrm{d}} /\left|\boldsymbol{v}_{\mathrm{d}}\right| .
$$

The gyromagnetic coupling vector $\boldsymbol{G}=(0,0, \mathcal{G})$ is given by

$$
\mathcal{G}=\int_{\text {unit cell }} \mathrm{d}^{2} r\left(\frac{\partial \hat{\mathbf{\Omega}}}{\partial x} \times \frac{\partial \hat{\mathbf{\Omega}}}{\partial y}\right) \cdot \hat{\mathbf{\Omega}}=4 \pi Q,
$$

where $Q(=+1)$ is the skyrmion number, and $\hat{\Omega}=\boldsymbol{M}(\mathbf{r}) / M$. On the other hand, the components of dissipative force tensor $\mathcal{D}$ are given by

$$
\mathcal{D}_{i j}=\int_{\text {unit cell }} \mathrm{d}^{2} r \partial_{i} \hat{\boldsymbol{\Omega}} \cdot \partial_{j} \hat{\mathbf{\Omega}}=\left\{\begin{array}{l}
\mathcal{D} \text { for }(i, j)=(x, x),(y, y), \\
0 \text { otherwise }
\end{array}\right.
$$

In the absence of impurities $\left(\boldsymbol{F}_{\text {pin }}=0\right)$, the drift velocity $\boldsymbol{v}_{\mathrm{d}}$ is derived from equation (3) as

$$
\begin{gathered}
\boldsymbol{v}_{\mathrm{d}}=\boldsymbol{v}_{\|}+\boldsymbol{v}_{\perp}, \\
\boldsymbol{v}_{\|}=\left(\frac{\beta}{\alpha}+\frac{\alpha-\beta}{\alpha^{3}(\mathcal{D} / \mathcal{G})^{2}+\alpha}\right) \boldsymbol{v}_{\mathrm{s}} \\
\boldsymbol{v}_{\perp}=\frac{(\alpha-\beta)(\mathcal{D} / \mathcal{G})}{\alpha^{2}(\mathcal{D} / \mathcal{G})^{2}+1}\left(\hat{z} \times \boldsymbol{v}_{\mathrm{s}}\right) .
\end{gathered}
$$

where $\boldsymbol{v}_{\|}$and $\boldsymbol{v}_{\|}$are the components of $\boldsymbol{v}_{\mathrm{d}}$ parallel and perpendicular to $\boldsymbol{v}_{\mathrm{s}}$, respectively. When $\alpha$ is small enough, we can neglect $\alpha^{3}$ in equation (8) and obtain $\boldsymbol{v}_{\|}=\boldsymbol{v}_{\mathrm{s}}$, which is consistent with our simulation showing the universal $j-v_{\|}$relation independent of $\beta$ with a realistic small value of $\alpha=0.04$. Equation (8) also suggests that deviation from the universal relation should show up in the extremely dissipative system with much larger $\alpha$. We verify below the validity of equations (8) and (9) in the cases of large $\alpha$ and $\beta$.

We first confirm the validity of Thiele's equation, that is, the static structure of the spin texture is kept during the motion. Figure 5a shows the comparison between the skyrmion structures at static case and steadily flowing case, where $r$ is the distance from the centre of the skyrmion and $M_{z}(r) / M$ is the $z$-component of the magnetization. (We interpolate the discrete lattice points by a smooth curve.) It is seen that almost no change occurs between the two cases, that is, there is no deformation of the SkX structure for finite $\alpha$ and $\beta$ during the translational motion, although it has some dependence on the external magnetic field as shown in Fig. 5b. This is not a trivial results as compared with the case of domain wall and HL where the spin tilting angle parallel to $\boldsymbol{v}_{\mathrm{s}}$ increases as $\left|\boldsymbol{v}_{\mathrm{s}}\right|$ increases as shown in Fig. $5 \mathrm{c}$, and the spin structure becomes oscillating when $\left|v_{s}\right|$ exceeds a critical value $\left|\boldsymbol{v}_{\mathrm{s}}\right|_{\mathrm{c}}$ where $\boldsymbol{v}_{\|}$deviates from the linear relation $v_{\|}=(\beta / \alpha) v_{\mathrm{d}}$. The latter case was not reached in the present simulations since the critical $\left|\boldsymbol{v}_{\mathrm{s}}\right|_{\mathrm{c}}$ is, in fact, three orders of magnitudes larger than the range of $j$ discussed here (see Methods).

In Fig. 6, we show the simulation data of $\boldsymbol{v}_{\|}$(a and b) and Hall angle $R=v_{\perp} / v_{\|}$(c and $\mathrm{d}$ ) as well as theoretical curves calculated from equations (8) and (9) as functions of $\alpha(\beta)$ for fixed $\beta(\alpha)$. Here we take $j=1.0 \times 10^{11} \mathrm{~A} \mathrm{~m}^{-2}$ and $B / J=0.015$, and $\mathcal{D}$ is evaluated as $\mathcal{D}=5.577 \pi$ (see Methods). We find that the simulated data coincide well with the theoretical curves. Also in Fig. 6e, we plot simulated $B$-dependence of $\mathcal{D}$ (see Methods) and Hall angle $R$ calculated using above estimated $\mathcal{D}$ as well as simulated $R$ for fixed $j=1.0 \times 10^{11} \mathrm{~A} \mathrm{~m}^{-2}$. The tendency that $R$ decreases as $B$ increases can be explained by the $B$-dependence of $\mathcal{D}$, but the discrepancy between the simulated and calculated values of $R$ becomes larger as $B$ is reduced. One possible reason is that the $r$-dependence of $M_{z}(r) / M$ is more rapid as $B$ is reduced, which gives the larger deviation from the continuum theory. The overall good agreement between the simulated and calculated results described above indicates that the theory developed in refs 31,32 based on Thiele's approach ${ }^{33}$ works well in the absence of impurities.

We next discuss the results in the presence of impurities by taking into account $\boldsymbol{F}_{\text {pin }} \neq 0$ in equations (3) and (4) ${ }^{31,32}$. Here we focus on the realistic value of $\alpha=0.04$ as in the case of Fig. 2 . As discussed above, the Hall angle $R$ gives important information, and hence we show in Fig. 7a the $j$-dependence of $R$ for $\beta, \alpha$, and $2 \alpha$ with the same condition as Fig. 2 . As $j$ increases, $R$ approaches to the asymptotic values expected from equations (8) and (9). On the other hand, it shows rapid increase due to the impurity effect as $j$ is decreased. This increase can be analysed by using equation (3) assuming small $\alpha$ and $\beta$. In the slow limit with low $j$, that is, $v_{\mathrm{d}}=\sqrt{v_{\|}^{2}+v_{\perp}^{2}} \sim 0$, we expect that the function $f\left(v_{\mathrm{d}} / v_{\text {pin }}\right)$ in equation (4) becomes unity. In this case, from the relationship $f \sim\left(v_{\mathrm{s}} v_{\perp}\right) /\left(v_{\mathrm{d}} v_{\text {pin }}\right)$ given in Supplementary of ref. 32, we obtain

$$
\frac{v_{\perp}}{v_{\mathrm{d}}}=\frac{R}{\sqrt{1+R^{2}}}=\frac{j_{\mathrm{c}}}{j},
$$

which indicates that $v_{\perp} / v_{\mathrm{d}}$ is proportional to $1 / j$. We indeed find such a linear relation between them in Fig. 7b. In addition, we can evaluate the magnitude of $j_{c}$ from the slope of this plot as $j_{\mathrm{c}}=3.51 \times 10^{9} \mathrm{~A} \mathrm{~m}^{-2}$. This value is an order of magnitude smaller than that for the HL as estimated in Fig. 2 and also as seen
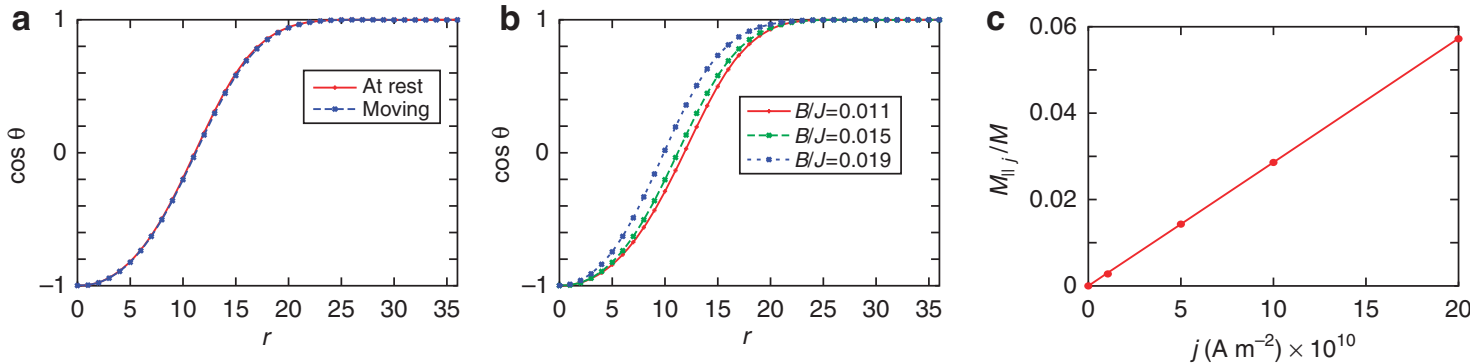

Figure 5 | Rigidity of Skyrmion spin texture. (a) Magnetic structures of static skyrmion $(j=0)$ and steadily flowing skyrmions $\left(j=1.0 \times 10^{11} \mathrm{Am} \mathrm{m}^{-2}\right)$ in the absence of impurities. The $z$-components of magnetization $M_{z} / M=\cos \theta$ for the two cases are plotted as functions of distance $r$ from the skyrmion centre for $\alpha=0.5, \beta=0.2$, and $B / J=0.015$, which shows their perfect coincidence. (b) Spin structures of skyrmion for different values of external magnetic field $B / J$, which shows that the skyrmion texture slightly varies when the value of $B / J$ changes. (c) Magnetization component $M_{\mathbf{j}} / M$ parallel to the electric current $\boldsymbol{j}$ for the $\mathrm{HL}$ texture during the current-driven translational motion is plotted as a function of $j$. 

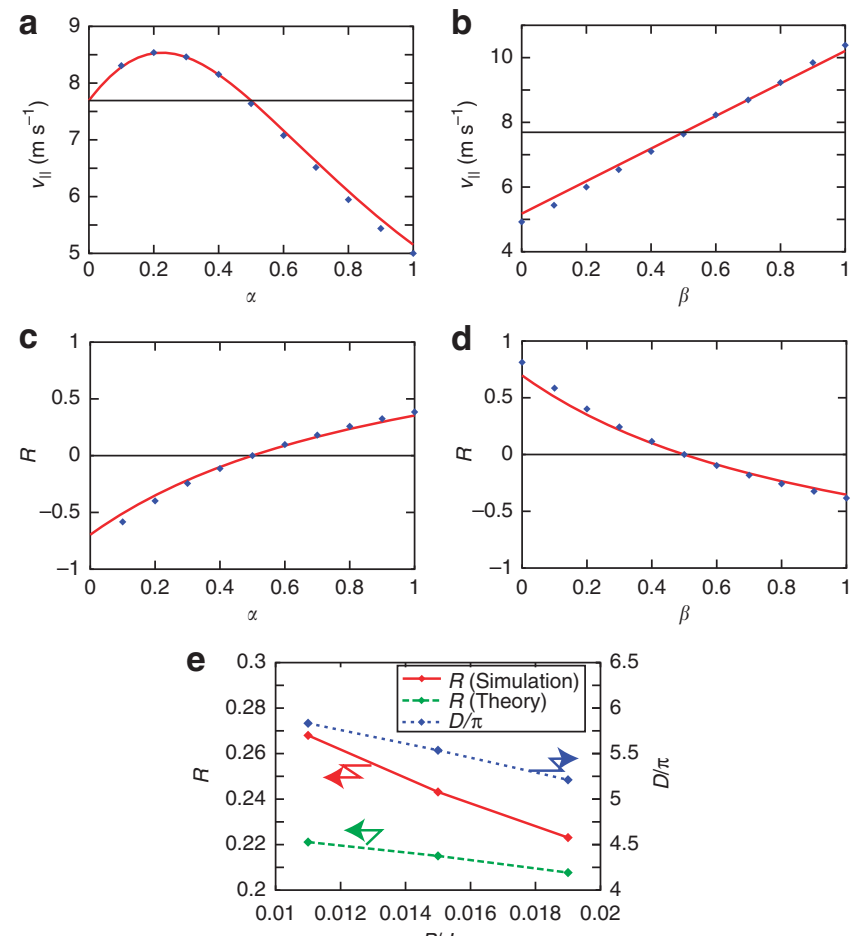

$B / J$

Figure 6 | Comparison between simulations and theory in the absence of impurities. Results of the simulations for the current-driven motion of skyrmion crystal in the absence of impurities are compared with the theory 31,32 based on Thiele's approach, which show excellent agreement. (a,c) Simulated $\alpha$-dependence of $v_{\|}$and Hall angle $R=v_{\perp} / v_{\|}$(blue dots) as well as theoretical curves of equations ( 8 ) and (9) (red lines) at fixed $\beta(=0.5)$. $(\mathbf{b}, \mathbf{d})$ Simulated $\beta$-dependence of $v_{\|}$and Hall angle $R$ (blue dots) as well as theoretical curves (red lines) at fixed $\alpha(=0.5)$. The simulations are done for $j=1.0 \times 10^{11} \mathrm{Am}^{-2}$ and $B / J=0.015$. (e) Simulated $B$-dependence of $D$ and $R$ as well as theoretically calculated $R$ using equations ( 8 ) and (9) from simulated $D$. The observed decreasing tendency of $R$ with increasing $B$ can be understood from the decrease of $D$ due to the deformation of skyrmion texuture under the magnetic field $\boldsymbol{B}$.

experimentally ${ }^{19}$. Fig. $7 \mathrm{c}, \mathrm{d}$ magnify the plots of $v_{\|}$and $v_{\mathrm{d}}^{2}=v_{\|}^{2}+v_{\perp}^{2}$ at low $j$, respectively. In Fig. $7 \mathrm{c}$, the decrease of $v_{\|}$ is observed below $j=1.0 \times 10^{10} \mathrm{~A} \mathrm{~m}^{-2}$, and the data can be extrapolated to zero at $j=j_{\mathrm{c}}=3.51 \times 10^{9} \mathrm{~A} \mathrm{~m}^{-2}$. More explicitly, solving equation (3) in the limit of $\alpha, \beta$, we obtain

$$
v_{\mathrm{d}}^{2}=\left(\frac{p a^{3}}{2 e M}\right)^{2}\left(j^{2}-j_{\mathrm{c}}^{2}\right),
$$

which is plotted in Fig. $7 \mathrm{~d}$ to compare with the simulation data. It is seen that the relation $v_{\mathrm{d}}^{2} \propto j^{2}-j_{\mathrm{c}}^{2}$ holds in the small $j$ region although the slope is slightly different between the simulation and equation (11). It is also reasonable that the impurity pinning effect is reduced as $j$ and $v_{\mathrm{d}}$ increase due to the motional narrowing effect. We also discuss the strength of pinning force. In the limit of $\beta \mathcal{D}^{\text {SkX }} \ll 4 \pi$, equation (3) for HL and that for SkX lead

$$
\frac{F_{\mathrm{pin}}^{\mathrm{SkX}}}{F_{\mathrm{pin}}^{\mathrm{HL}}}=\frac{1}{\pi \beta} \frac{j_{\mathrm{c}}^{\mathrm{SkX}}}{j_{\mathrm{c}}^{\mathrm{HL}}}
$$

as $\mathcal{G}^{\mathrm{SkX}}=4 \pi$ and $\mathcal{D}^{\mathrm{HL}}=4 \pi^{2}$. Our simulation (in the case of $\alpha=\beta=0.04$ ) gives $F_{\text {pin }}^{\mathrm{SkX}} / F_{\text {pin }}^{\mathrm{HL}}=0.56$. This weak $F_{\text {pin }}^{\mathrm{SkX}}$ compared with $F_{\text {pin }}^{\mathrm{HL}}$ is due to deformations of the skyrmion triangular lattice
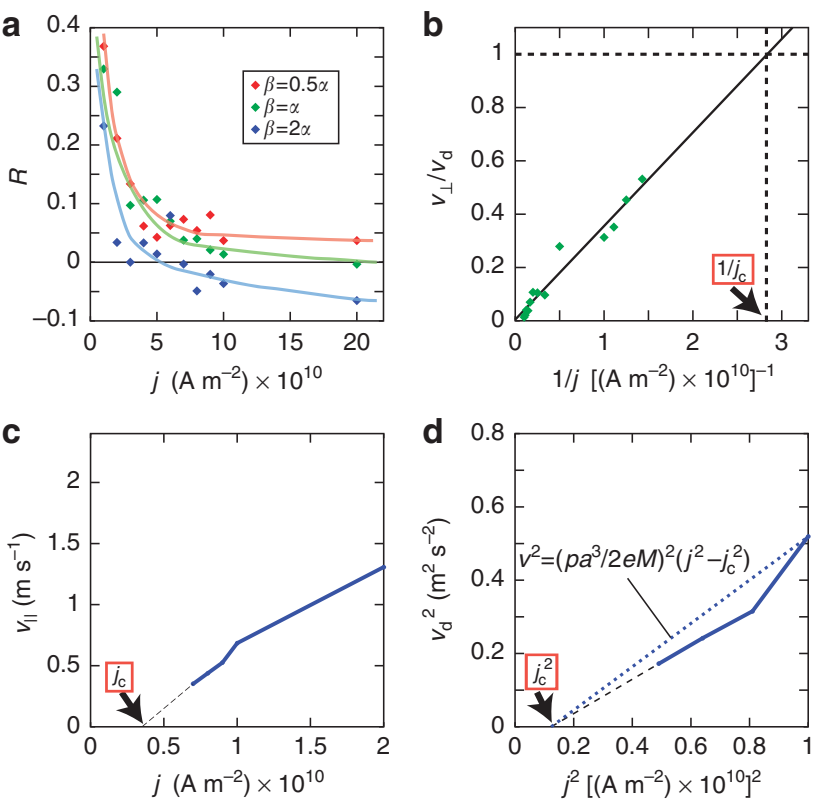

Figure 7 | Comparison between simulations and theory in the presence of impurities. Results of the simulations for the current-driven motion of skyrmion crystal in the presence of impurities are compared with the theory 31,32 based on Thiele's approach. (a) Simulated $j$-dependence of Hall angle $R=v_{\perp} / v_{\|}$for several values of $\beta$, that is, $\beta=0.5 \alpha, \alpha$ and $2 \alpha$ with $\alpha=0.04$ in the presence of impurities $(x=0.1 \%)$. Here, the solid lines are guides for the eyes. (b) Theoretically predicted linear relation equation (10) (solid line) between $v_{\perp} / v_{d}$ and $j_{d} / j$ in the slow limit with low $j$. The relation is indeed reproduced by the simulation (dots). The plot gives $j_{\mathrm{c}}=3.51 \times 10^{9} \mathrm{~A} \mathrm{~m}^{-2}$. (c) Simulated $j-v_{\|}$plot in the small $j$ region is magnified. The dashed line is an extrapolation to $j_{c}$ for $v_{\|}=0$ estimated in Fig. 7b. (d) Theoretically predicted linear relation equation (11) (dotted line) between $v_{\mathrm{d}}^{2}$ and $j^{2}$ in the limit of low $j$ and small $\alpha$ and $\beta$. The simulated data of $v_{\mathrm{d}}^{2}$ is also plotted as a function of $j^{2}$ (dots and solid line). The dashed line indicates an extrapolation to $j_{\mathrm{c}}^{2}$ for $v_{\mathrm{d}}=0$ estimated in Fig. $7 \mathrm{~b}$.

and individual skyrmions to avoid the pinning centres as shown above.

It is established above that Thiele's approach for the translational motion with assumed rigid spin texture works well even in the presence of impurities for the centre of mass motion of SkX. The apporach works well as far as we discuss the quantities averaged over space and time such as velocities and Hall angle although the triangular lattice of skyrmions as well as the individual skyrmions are considerably deformed during the motion from their original shapes so as to avoid the impurities.

To conclude we have discovered the novel universal currentvelocity relation of the skyrmion motion in chiral-lattice magnets which is in sharp contrast to that of HL or the ferromagnetic domain wall. It is insensitive to the Gilbert damping, the nonadiabatic effect, and the impurity pinning. The critical current density $j_{c}$ for the SkX motion is also of the order of magnitude smaller than that for the HL motion. Therefore, the SkX is an ideal spin texture to achieve the electric-current control via the spin-transfer-torque mechanism with ultralow current density. Our theory provides a basis to utilize skyrmions as information carriers with low energy cost.

\section{Methods}

Numerical simulation of LLG equation. We use the fourth-order Runge-Kutta method to solve the LLG equation. The simulations are done for a system of $288 \times 288$ sites where the periodic boundary condition is imposed. The initial spin 
configurations are obtained by the Monte-Carlo thermalization and by further relaxing them at $j=0$ in the LLG simulation. After the sufficient convergence of the spin configurations, we switch on a steady electric current and observe the spin dynamics. In the HL state, the direction of electric current is set to be parallel to its propagation vector. The natural units of time $t$ and current density $j$ are $\tau \equiv \hbar / J$ and $\kappa \equiv \frac{2 e M I}{p a^{2} \hbar}$, respectively. With a typical lattice constant $a=5 \AA$, spin-polarization $p=0.2$ and the magnitude of local magnetic moment $M=1$, the values of $\tau$ and $\kappa$ become $\tau \simeq 6.5 \times 10^{-13} \mathrm{~s}$ and $\kappa \simeq 1.0 \times 10^{13} \mathrm{~A} \mathrm{~m}^{-2}$. We take these values to convert the units of simulated current density, velocity and time.

Effects of magnetic dipole interaction. In usual ferromagnets with uniaxial anisotropy, the lattice of magnetic bubbles can be formed through the competition among the exchange energy, the anisotropy energy and magnetostatic energy. The relationship between the lattice spacing $d$ of the bubbles and the sample thickness $l$ can be derived as $d^{2}=\frac{2 \sqrt{2 \pi} \gamma l}{4.74 \times 10^{4} \mathrm{NI}_{\mathrm{s}}}$ by minimizing the total energy per unit area, $E=4.74 \times 10^{4} \mathrm{NI}_{\mathrm{s}} d+\gamma 2 \sqrt{2 \pi} l / d$ (ref. 20). Here, $I_{\mathrm{s}}(=1)$ is the saturation moment, $\gamma=4 \sqrt{A K}, K$ is the strength of uniaxial anisotropy, and $A=J M^{2} / a$. We adopt $\gamma=1.6 \times 10^{-3}$ for the iron [100]-plane as a typical value of $\gamma$. If we assume that the demagnetizing coefficient $N$ is unity, the sample thickness, which gives $d \sim 10 \mathrm{~nm}$, is estimated to be $l \sim 1 \mathrm{~nm}$. In the real materials, the value of $N$ sensitively depends on the sample shape and thickness, and it is usually orders of magnitude smaller than unity. Thus, the required thickness is much smaller than $1 \mathrm{~nm}$.

Calculation of the dissipative force tensor $\boldsymbol{D}$ for SkX. The dissipative force tensor $D$ defined in equation (6) can be calculated for $\mathrm{SkX}$ by focusing on a single skyrmion with its centre at $\boldsymbol{r}=\mathbf{0}$ because in the region between the skyrmions, the spins are pointing in the $z$-direction. The symmetry of the spin configuration around $\boldsymbol{r}=\mathbf{0}$ leads to

$$
\mathcal{D}_{i j}=\mathcal{D} \delta_{i j}
$$

with

$$
\mathcal{D}=\pi \int_{0}^{R_{s}} r \mathrm{~d} r\left[\left(\frac{\mathrm{d} \theta(r)}{\mathrm{d} r}\right)^{2}+\frac{\sin ^{2} \theta(r)}{r^{2}}\right],
$$

where $r=|r|$ is the distance from a skyrmion core, $R_{\mathrm{s}}=\mathrm{Na}$ ( $a$ is the lattice constant) is the radius of skyrmion, and $\cos \theta(r)=M_{z}(r) / M . M(r)$ is discretized in our simulation, and the following simple discrete summation is employed:

$$
\mathcal{D}=\pi \sum_{i=1}^{N} i\left[\left(\frac{\theta(i+1)-\theta(i-1)}{2}\right)^{2}+\frac{\sin ^{2} \theta(i)}{i^{2}}\right] .
$$

The simulated $r$-dependence of $\theta(i)$ with $r=i a$ is shown in Fig. 5a,b for several values of $B$, and they give the $B$-dependence of $\mathcal{D}$ in Fig. $6 \mathrm{e}$.

Critical current density of the intrinsic pinning for $\mathbf{H L}$. In the case of $\mathrm{HL}$, the Magnus force does not work, that is, $\mathcal{G}=0$ in Thiele's equation. Therefore, in the absence of impurity, $v_{\mathrm{d}}=(\beta / \alpha) v_{\mathrm{s}}$, and especially $v_{\mathrm{d}}=0$ when $\beta=0$, which is called the intrinsic pinning. However, Thiele's equation is valid only below a certain current density $j_{\mathrm{c}}^{\mathrm{i}}$, above which the spin configuration becomes oscillatory timedependent. The value of $j_{c}^{i}$ can be estimated by considering the strength of the easyplane spin anisotropy, which is generated by the DM interaction in the chirallattice magnets. We estimate strength of the anisotropy and $j_{c}^{i}$. We assume the helical structure with rotating angle $\theta$ and tilting angle $\phi$. Then the energy per spin from the DM interaction, $h_{\mathrm{DM}}$, becomes

$$
\begin{aligned}
h_{\mathrm{DM}}= & \sqrt{2}|D \sin \theta| \sin ^{2} \phi+\text { constant } \\
& \equiv K_{\perp} \sin ^{2} \phi+\text { constant. }
\end{aligned}
$$

Using the above-derived relation $K_{\perp}=\sqrt{2}|D \sin \theta|$, we can derive the expression of $j_{c}^{i}$, and can estimate its typical value ${ }^{34}$

$$
\begin{gathered}
j_{\mathrm{c}}^{\mathrm{i}}=\frac{e M^{2} \lambda K_{\perp}}{\hbar a^{3} p} \\
\simeq 1 \times 10^{13} \mathrm{Am}^{-2},
\end{gathered}
$$

where $\lambda$ is the periodicity of HL. This value is much larger than the current density of our interest in the present paper.

Expression of $v_{,} v_{\|}$and $v_{\perp}$ of skyrmion in the presence of impurities. The function $f$ in the equation (4) is $\sim 1$ for small $v_{\mathrm{s}}$ (or $j$ ), and in this case we can derive the explicit expression for $v_{\mathrm{d}}, v_{\|}$, and $v_{\|}$of skyrmion:

$$
\begin{gathered}
v_{\mathrm{d}}=\frac{\sqrt{(\alpha \mathcal{D} A)^{2}+\left(\alpha^{2} \mathcal{D}^{2}+\mathcal{G}^{2}\right)\left[\left(\beta^{2} \mathcal{D}^{2}+\mathcal{G}^{2}\right) v_{\mathrm{s}}^{2}-A^{2}\right]}}{\alpha^{2} \mathcal{D}^{2}+\mathcal{G}^{2}}, \\
v_{\|}=\frac{v_{\mathrm{s}}}{\left(\alpha \mathcal{D}+A / v_{\mathrm{d}}\right)^{2}+\mathcal{G}^{2}} \times\left[\left(\alpha \mathcal{D}+A / v_{\mathrm{d}}\right) \beta \mathcal{D}+\mathcal{G}^{2}\right], \\
v_{\perp}=\frac{v_{\mathrm{s}}}{\left(\alpha \mathcal{D}+A / v_{\mathrm{d}}\right)^{2}+\mathcal{G}^{2}} \times\left[\left(\alpha \mathcal{D}+A / v_{\mathrm{d}}\right) \mathcal{G}-\beta \mathcal{D} \mathcal{G}\right],
\end{gathered}
$$

where $A \equiv 4 \pi v_{\text {pin }}$. In the limit of $\alpha, \beta \rightarrow 0$, we obtain equations (10) and (11) from these equations.

\section{References}

1. Slonczewski, J. C. Current-driven excitation of magnetic multilayers. J. Magn. Magn. Mater. 159, L1-L7 (1996).

2. Berger, L. Emission of spin waves by a magnetic multilayer traversed by a current. Phys. Rev. B 54, 9353-9358 (1996).

3. Maekawa, S. Concepts in Spin Electronics. Ch 7 (Oxford University Press, Oxford, 2006).

4. Parkin, S. S. P., Hayashi, M. \& Thomas, L. Magnetic domain-wall racetrack memory. Science 320, 190-194 (2008).

5. Yamanouchi, M., Chiba, D., Matsukura, F. \& Ohno, H. Current-induced domain-wall switching in a ferromagnetic semiconductor structure. Nature 428, 539-542 (2004).

6. Tatara, G. \& Kohno, H. Theory of current-driven domain wall motion: spin transfer versus momentum transfer. Phys. Rev. Lett. 92, 086601 (2004).

7. Tatara, G. et al. Threshold current of domain wall motion under extrinsic pinning, $\beta$-term and non-adiabaticity. J. Phys. Soc. 75, 064708 (2006).

8. Brataas, A., Kent, A. D. \& Ohno, H. Current-induced torques in magnetic materials. Nat. Mater. 11, 372-381 (2012).

9. Barnes, S. E. \& Maekawa, S. Current-spin coupling for ferromagnetic domain walls in fine wires. Phys. Rev. Lett. 95, 107204 (2005).

10. Skyrme, T. H. R. A unified field theory of mesons and baryons. Nuc. Phys. 31, 556-569 (1962).

11. Bogdanov, A. N. \& Yablonskii, D. A. Thermodynamically stable 'vortices' in magnetically ordered crystals. The mixed state of magnets. Sov. Phys. JETP 68, 101-103 (1989).

12. Rößler, U. K., Bogdanov, A. N. \& Pfleiderer, C. Spontaneous skyrmion ground states in magnetic metals. Nature 442, 797-801 (2006).

13. Mühlbauer, S. et al. Skyrmion lattice in a chiral magnet. Science 323, 915-919 (2009).

14. Yi, S. D., Onoda, S., Nagaosa, N. \& Han, J. H. Skyrmions and anomalous Hall effect in a Dzyaloshinskii-Moriya spiral magnet. Phys. Rev. B 80, 054416 (2009).

15. Butenko, A. B., Leonov, A. A., Rößler, U. K. \& Bogdanov, A. N. Stabilization of skyrmion textures by uniaxial distortions in noncentrosymmetric cubic helimagnets. Phys. Rev. B 82, 052403 (2010).

16. Yu, X. Z. et al. Real-space observation of a two-dimensional skyrmion crystal. Nature 465, 901-904 (2010).

17. Yu, X. Z. et al. Near room-temperature formation of a skyrmion crystal in thinfilms of the helimagnet FeGe. Nat. Mater. 10, 106-109 (2011).

18. Jonietz, F. et al. Spin transfer torques in $\mathrm{MnSi}$ at ultralow current densities. Science 330, 1648-1651 (2010).

19. Yu, X. Z. et al. Skyrmion flow near room temperature in an ultralow current density. Nat. Commun. 3, 988 (2012).

20. Kittel, C. Physical Theory of Ferromagnetic Domains. Rev. Mod. Phys. 21, 541-583 (1949).

21. Mizukami, S., Ando, Y. \& Miyazaki, T. The study on ferromagnetic resonance linewidth for $\mathrm{NM} / 80 \mathrm{NiFe} / \mathrm{NM}(\mathrm{NM}=\mathrm{Cu}, \mathrm{Ta}, \mathrm{Pd}$ and $\mathrm{Pt})$ Films. Jpn. J. Appl. Phys. 40, 580-585 (2001).

22. Mizukami, S. et al. Gilbert damping in perpendicularly magnetized $\mathrm{Pt} / \mathrm{Co} / \mathrm{Pt}$ films investigated by all-optical pump-probe technique. Appl. Phys. Lett. 96, 152502 (2010).

23. Oogane, M. et al. Magnetic damping in ferromagnetic thin film. J. Phys. Soc. Jpn. 45, 3889-3891 (2006)

24. Oogane, M. et al. Gilbert magnetic damping constant of epitaxially grown Cobased Heusler alloy thin films. Appl. Phys. Lett. 96, 252501 (2010).

25. Nemec, P. et al.Establishing micromagnetic parameters of ferromagnetic semiconductor (Ga,Mn)As, Preprint at http://arXiv.org/abs/1207.0310 (2012).

26. Bazaliy, B., Jones, B. A. \& Zhang, S. C. Modification of the Landau-Lifshitz equation in the presence of a spin-polarized current in colossal- and giantmagnetoresistive materials. Phys. Rev. B 57, R3213 (1998).

27. Zang, J., Mostovoy, M., Han, J. H. \& Nagaosa, N. Dynamics of skyrmion crystals in metallic thin films. Phys. Rev. Lett. 107, 136804 (2011).

28. Fukuyama, H. \& Lee, P. A. Dynamics of the charge-density wave. I. Impurity pinning in a single chain. Phys. Rev. B 17, 535-541 (1978).

29. Lee, P. A. \& Rice, T. M. Electric field depinning of charge density waves. Phys. Rev. B 19, 3970-3980 (1979). 
30. Georgii, R. et al. Turn-key module for neutron scattering with sub-micro-eV resolution. Appl. Phys. Lett. 98, 073505 (2011).

31. Everschor, K. et al. Rotating skyrmion lattices by spin torques and field or temperature gradients. Phys. Rev. B 86, 054432 (2012).

32. Schulz, T. et al. Emergent electrodynamics of skyrmions in a chiral magnet. Nat. Phys. 8, 301-304 (2012).

33. Thiele, A. A. Steady-state motion of magnetic domains. Phys. Rev. Lett. 30, 230-233 (1972).

34. Tatara, G., Kohno, H. \& Shibata, J. Microscopic approach to current-driven domain wall dynamics. Phys. Rep. 468, 213-301 (2008).

\section{Acknowledgements}

The authors are grateful for insightful discussions with Y. Tokura and X.Z. Yu, and for the design of Fig. 1 with M. Ishida. This work was supported by Grant-in-Aids for Scientific Research (No. 24224009) from the Ministry of Education, Culture, Sports, Science and Technology (MEXT) of Japan, and by Funding Programme for World-
Leading Innovative R and D on Science and Technology (FIRST Programme). M.M. was supported by the G-COE Programme 'Physical Sciences Frontier' from MEXT of Japan.

\section{Author contributions}

J.I. performed the numerical calculations. J.I., M.M. and N.N. contribute in analysing the data and writing the paper.

\section{Additional information}

Supplementary Information accompanies this paper on http://www.nature.com/ naturecommunications

Competing financial interests: The authors declare no competing financial interests.

Reprints and permission information is available online at http://npg.nature.com/ reprintsandpermissions/

How to cite this article: Iwasaki, J. et al. Universal current-velocity relation of skyrmion motion in chiral magnets. Nat. Commun. 4:1463 doi: 10.1038/ncomms2442 (2013). 